\title{
Implementación del cómputo en la nube: análisis de los beneficios reportados en empresas del sur de sonora del sector productivo y de servicios \\ Cloud computing implementation: analysis of benefits reported by productive sector and services companies of south sonora
}

\author{
Francisco Alan Espinoza Zallas \\ Universidad Estatal de Sonora \\ alanez@outlook.com \\ Sergio Carlos Blanco Guzmán \\ Universidad Estatal de Sonora \\ sergio.blanco@ues.mx \\ Margarita Soto Rodríguez \\ Universidad Estatal de Sonora \\ maguysoto@hotmail.com
}

\section{RESUMEN}

En la actualidad el software ha cumplido funciones muy importantes que llevan a facilitar procesos y realizar actividades con un mejor desempeño y rapidez, siendo innumerables los beneficios obtenidos de su buen uso. Las organizaciones del siglo XXI tienen algunas problemáticas, una de ellas es que deben reducir gastos, para lo cual requieren optimizar sus procesos operativos e implementar controles para crear mejoras y detectar focos rojos.

Por esta razón, es necesario hacer uso de los sistemas de información, mismos que sirven para crear estos controles, y lograr ayuda para la toma de decisiones en las organizaciones. Además, se aprovecha el internet para así poder crear un vínculo que hará posible el acceso a la información de las operaciones de sus empresas por parte de los directivos, desde cualquier lugar del mundo, solo con tener acceso a la Red Global.

Palabras clave: tecnologías de información, sistemas de información en la nube, software. 


\section{Abstract}

Nowadays software has fulfilled important functions that lead to simplify processes and activities with better performance and speed, being innumerable benefits obtained from good use. The 21 st century organizations have some problems, one of which is that it should reduce expenditure, which requires optimize its business processes and implement controls to create improvements and detect red flags.

For this reason, it is necessary to make use of information systems, same that are used to create these controls, and support for decision-making in organizations. In addition, the internet takes advantage to be able to create a link that will make it possible to access to the information of the operations of their companies by managers, from anywhere in the world, only to have access to the Global Network.

Key Words: information technology, information systems in the cloud, software

Fecha recepción: Marzo $2013 \quad$ Fecha aceptación: Mayo 2013

\section{Introducción}

La mayoría de las empresas necesitan de Software que les simplifique la operatividad empresarial. Con la llegada del Internet a la mayoría de las organizaciones, estas tienen una nueva opción: el uso de Software de Control en la nube, lo cual es una opción viable que ayuda a las organizaciones a minimizar costos para llevar controles operativos a través de software y también a destinar menos dinero en la implementación de estos sistemas de información en la empresa, logrando ahorrar costos en compra e instalación de servidores y no tener la necesidad de absorber los costos de mantenimiento de hardware, como comúnmente sucede al tener software estacional. 


\section{Planteamiento del problema}

El mundo en el que vivimos es de cambios constantes; quien tiene la información, tiene ventaja sobre quien no cuenta con ella, y quien tiene la información en tiempo real y con disponibilidad de 24/7, está un paso adelante de quien solo cuenta con la información. Sin embargo, para que las organizaciones tengan la información necesaria en tiempo real, necesitan de las tecnologías de información, las cuales forman parte de la mayoría de las empresas de hoy en día. Es común que cada empresa que nace o existe, tenga la necesidad de tener por lo menos una computadora, considerando que ayudará a impulsar el desarrollo integral de la organización. A pesar de la existencia de gran variedad y cantidad de software comercial de apoyo gerencial, en muchas ocasiones solo se utiliza la computadora como almacén de datos; contemplando en ella el registro de ventas, compras, inventario y recursos humanos, en su mayoría con el propósito de tomar decisiones en algún momento dado.

En la presente investigación se denotan los beneficios encontrados al implementar sistemas de información en la nube que han obtenido las empresas del sur de Sonora. En ella se plasma la observación directa de los beneficios que obtienen los miembros de las empresas, entre los que están directivos, administrativos, auxiliares y demás personal. Los administrativos son los que más necesitan de estos servicios y los que obtienen mayor provecho al implementarlos.

Los beneficios encontrados con mayor frecuencia son: compartir la información, disminuir duplicidad de archivos, implementar controles operativos, tener acceso a los datos de manera remota y desde dispositivos móviles, tener mayor control de inventarios y reducir 
Revista Iberoamericana de las Ciencias Computacionales e Informática ISSN: 2007-9915

costos de implementación y desarrollo. Las anteriores representan las ventajas competitivas que pueden tener las empresas con la implementación de tecnologías de información en la nube.

Para la realización de la investigación se partió de la siguiente pregunta:

¿Cuáles son los principales beneficios de la implementación de software de control operativo en las organizaciones del sur de Sonora?

La presente investigación se realizó en organizaciones del sur de Sonora.

\section{Objetivo}

Conocer los principales beneficios de la gestión de control operativo en la nube en la región sur de Sonora.

\section{Antecedentes}

En el caso de los docentes, las tecnologías ponen a su disposición diversos recursos digitales: software, documentos, página web, etcétera.; facilitan la participación en redes de docentes y apoyan el trabajo de proyectos en forma colaborativa con otros centros educativos (Collins 1998). Otro autor, Cisneros F. (2009), en su investigación nos muestra que el éxito para la innovación tecnológica en el ámbito educativo depende en gran medida de la actuación del maestro, la cual es determinada por su formación en las nuevas tecnologías. Por otra parte, el estudio de Martínez J. L. (2008), refleja la carencia de proyectos formativos y de capacitación en el uso de las TIC.

Con respecto a la utilización de las nuevas tecnologías, se han desarrollado investigaciones que demuestran las ventajas de su utilización en las organizaciones. Las tecnologías de 
Revista Iberoamericana de las Ciencias Computacionales e Informática ISSN: 2007-9915 información pueden ser una ventaja competitiva; con relación a esto, SOMERS y NELSON (2002) consideran que la estrategia de negocio está relacionada con la organización, la tecnología y las decisiones estratégicas de las operaciones (organización, tecnología, calidad y localización, gestión del talento humano, etcétera) que soportan la estrategia empresarial. La clave en el desarrollo de las capacidades de los sistemas de información (SI) de la empresa es saber articular la función estratégica y la gestión del propio SI mediante la definición de una arquitectura de TI apropiada y una estructura de control que faciliten su desarrollo y explotación (Stratman y Aleda, 2002).

En otra investigación, Paulo André da (2010) menciona que los sistemas ERP son un apoyo a la gerencia, Conceição Menezes nos menciona que los sistemas de planificación de los recursos empresariales (Enterprise ResourcesPlanning, ERP) facilitan la integración y la optimización de los procesos de negocio mediante la aplicación de Tecnologías de la Información.

Hong y Kim, 2002, hace mención de que el entorno y la operativa de las pequeñas y medianas empresas (PYMES) son distintos de los de las grandes empresas y esto afecta a la implantación de un ERP. Aunque se ha investigado mucho su implantación en grandes empresas, existe menos información sobre el impacto de los ERP en las PYMES y sobre cómo se obtienen beneficios tras su puesta en marcha; tampoco se conocen bien los factores que han dificultado o facilitado un rendimiento óptimo. Los factores que deben gestionarse en esta fase son: la definición de los objetivos estratégicos del ERP, el compromiso de la dirección y la disposición al cambio estructural y cultural (Motwany et al., 2005). Rajagopal (2002) la denomina fase de "adopción”, propone un modelo compuesto de seis 
Revista Iberoamericana de las Ciencias Computacionales e Informática ISSN: 2007-9915

fases y sugiere, como actividades típicas, la decisión de inversión, el análisis de costebeneficio y la elección de la tecnología y su proveedor.

Seleccionar el ERP idóneo exige tener en cuenta las necesidades derivadas de la estrategia (información obtenida de los clientes, proveedores y competidores). Además, las características de un ERP que facilitan la reingeniería de procesos de negocio son: el alcance, la capacidad de configuración del sistema y su capacidad de integración; pudiendo aplicarse diferentes combinaciones de las perspectivas BPR (Business ProcessReengineering, Reingeniería de los procesos de negocio) y ERP con vistas a lograr la integración requerida en procesos interfuncionales (Koch, 2001; Ugrin, 2009).

\section{Marco teórico}

\section{a. Computadoras}

Una computadora es un dispositivo electrónico que te permite procesar datos para poder convertirlos en información de utilidad. Las computadoras son capaces de ejecutar cálculos y tomar decisiones lógicas mucho más rápido que un ser humano. Toda computadora está compuesta de hardware y software.

b. Hardware

Es todo lo que puedes tocar de una computadora, son todos los dispositivos electrónicos interconectados.

En esencia, una computadora es un dispositivo que da entrada, procesa y almacena datos y produce salida; todo ello de acuerdo con una serie de instrucciones almacenadas que se conocen como programas de computación. 
Revista Iberoamericana de las Ciencias Computacionales e Informática ISSN: 2007-9915

La utilidad de las computadoras radica en que te ayudan a procesar grandes volúmenes de información en poco tiempo, puedes almacenar de manera permanente información para tomar mejores decisiones y con un índice de error menor.

c. Software

El software se refiere a los programas y datos almacenados en una computadora. En otras palabras, son las instrucciones responsables de que el hardware (la máquina) realice su tarea.

El lenguaje utilizado por el software, para comunicarse con el hardware, es de tipo binario, viene en forma de instrucciones, las cuales son ejecutadas por cada una de las partes del hardware (monitor, mouse, teclado, impresora, CPU, CD-ROM, disco duro, etcétera).

El software puede dividirse en tres categorías básicas:

Software del sistema, software de aplicación, software de programación, software del sistema. Es el software básico o sistema operativo. Es un conjunto de programas que controlan los trabajos de la computadora. Se encarga de administrar y asignar los recursos de hardware.

Software de aplicación: son los programas que controlan y dirige las distintas tareas que realizan las computadoras, creando un ambiente amigable entre el pc y el usuario. Llevan a cabo tareas de tratamiento de textos, gestión de bases de datos y similares.

Software de programación: son los lenguajes de programación, intérpretes, compiladores y aplicaciones similares utilizadas por los desarrolladores de sistemas. 
Revista Iberoamericana de las Ciencias Computacionales e Informática ISSN: 2007-9915

d. Las nuevas tecnologías

Las nuevas tecnologías de la información y las comunicaciones tienen, día a día, una mayor presencia en todos los aspectos de la vida laboral y personal (Schoemaker y Jonker, 2005; Breivold et al., 2012), ofreciendo un nuevo espacio de innovación en ámbitos como la industria, los servicios, la salud, la administración, el comercio y la educación.

Se consideran Tecnologías de la Información y Comunicación tanto al conjunto de herramientas relacionadas con la transmisión, procesamiento, almacenamiento y mantenimiento digitalizado de información, como al conjunto de procesos y productos derivados de las nuevas herramientas (es decir, tanto al hardware como al software), (Longley y Shain, 1985).

El incluir la palabra "Nuevas" se debe a que existen otras tecnologías anteriores -como es el caso del teléfono-, que también se refieren a la información y las comunicaciones, pero que no quedarían recogidas dentro del concepto de NTIC. En otras palabras, las NTIC tratan sobre el uso de hardware (computadora, Smartphone, etcétera) y software (diferentes aplicaciones como ERP, CRM, etcétera) que permiten transformar, almacenar, gestionar, proteger, difundir y localizar los datos necesarios para cualquier actividad humana (ITAA, Adelman, 2000).

\section{e. Tecnología}

Una tecnología puede ser hardware y software. Un sistema computacional es el conjunto de tecnologías que interactúan entre sí. El sistema computacional toma datos del medio ambiente (entrada), analiza y procesa estos datos, los almacena (proceso) y, finalmente, 
Revista Iberoamericana de las Ciencias Computacionales e Informática ISSN: 2007-9915

despliega como producto final la información (salida), la cual puede ser usada para realizar una acción en ese medio ambiente (retroalimentación).

\section{f. Las organizaciones y el internet}

La amplia utilización de las NTIC en el mundo ha traído como consecuencia un importante cambio en la economía mundial, particularmente en los países más industrializados, sumando a los factores tradicionales de producción para la generación de riquezas, un nuevo factor que resulta estratégico: el conocimiento (Craig, 2007). Esta situación obliga a nuestras economías y, en última instancia, a nuestras empresas a desarrollar productos y servicios de mayor valor añadido (Pérez y Dressler, 2007), evolucionando hacia modelos en los que la importancia en los procesos industriales es reemplazada por la relevancia del procesamiento de a información y el conocimiento como claves económicas (Schoemaker y Jonker, 2005).

De esta forma, en el nuevo entorno la competitividad de las empresas se ve comprometida por dos aspectos interrelacionados: el primero, un uso intensivo y racional de las tecnologías de la información y las comunicaciones, que cree valor para la organización y favorezca el segundo aspecto, el conocimiento, recurso fundamental de las empresas (Lueg, 2001). El hecho de que los profesionales de la organización tengan mayor información y conocimiento disponible es la clave para conseguir una ventaja competitiva sostenible en el tiempo (Shoemaker, 2001). Es por eso que ya no solo se habla de la "sociedad de la información", sino también de la "sociedad del conocimiento".

g. Conceptos básicos de la computación en la nube (cloud computing) 
Revista Iberoamericana de las Ciencias Computacionales e Informática ISSN: 2007-9915

Existen varias definiciones, unas sencillas y otras más técnicas. Su nombre viene de la forma que ha sido representada en diagramas, a la apariencia de computadoras conectadas entre sí. Pudo haber sido "la red”, pero se le nombró "la nube” y según (Gutiérrez, 2010): la nube es un término que se refiere a la forma de una red de computadoras (Grid Computing), como proveedor de servicios de software y datos, donde Grid Computing es un conjunto de computadoras conectadas entre sí que comparten recursos. Es un modelo de procesamiento que permite resultados más rápidos que los que se podrían lograr con un modelo clienteservidor, en el cual existe un servidor central (MainFrame) que concentra todas las transacciones y cuenta con toda la información y datos, así como con la lógica del negocio. Por otro lado, cuando el cliente (usuario) hace una petición a la Nube es atendido por muchas computadoras logrando así tiempos de respuesta mucho más rápidos y una mayor disponibilidad puesto que el sistema no depende de un solo servidor.

National Institute of Standards and Technology del Departamento de Comercio del Gobierno Federal de los Estados Unidos define cloud computing de la siguiente manera: "El cloud computing es un modelo de computación que permite, desde cualquier lugar y de un modo práctico, el acceso bajo demanda y a través de la red a un conjunto de recursos informáticos compartidos y configurables (por ejemplo: redes, servidores, equipos de almacenamiento, aplicaciones y servicios), los cuales pueden ser rápidamente aprovisionados y liberados tras esfuerzos de gestión mínimos y poca interacción con el proveedor del servicio". (NIST, 2011).

Tratando este tema con un lenguaje más coloquial, Plummer (2012) la define de la siguiente manera: 
Revista Iberoamericana de las Ciencias Computacionales e Informática ISSN: 2007-9915

"La computación en nube significa que alguien más se encarga de administrar las computadoras y el software, mientras tú solo usas lo que te entregan y te concentras en valorar la calidad del servicio."

Añadido a esta definición, Plummer invita a pensar que cloud computing son recursos que cualquiera puede utilizar sin ser propietarios de ellos. Todos pagan una parte del costo, por lo que disminuye el gasto total para todos.

\section{Método}

Para realizar la investigación se utilizó el estudio de casos, que es un diseño de investigación que afronta la realidad mediante un análisis detallado de sus elementos y la interacción que se produce entre ellos y su contexto, para llegar mediante un proceso de síntesis a la búsqueda del significado y la toma de decisiones sobre el caso.

Bell (2002) plantea que el investigador de estudios de caso se propone identificar las características y mostrar cómo afectan a la puesta en práctica de sistemas e influyen en la forma de funcionar de la institución.

Se consideró el método apropiado de acuerdo al objetivo de la investigación, principalmente si se obtuvo la información mediante entrevistas semiestructuradas a personal de organizaciones de la región. Como complemento, en la investigación se realizaron varias visitas para obtener información mediante observaciones sobre la utilización de nuevas tecnologías. 
Revista Iberoamericana de las Ciencias Computacionales e Informática ISSN: 2007-9915

Para llevar a cabo la investigación se entrevistaron a diez organizaciones privadas y públicas ubicadas en la Ciudad de Navojoa. Las organizaciones que se tomaron en cuenta para el estudio fueron las siguientes:

Clarvi, Contraloría del Estado de Sonora, Kowi, Bioderpack, CMIC Navojoa, ITC Sonora, XXI Construcciones, Hospital San José Navojoa, Cavar, Exim Alimentos.

\section{Análisis e interpretación de resultados}

Con el análisis de las entrevistas y la observación directa a las personas que utilizan los sistemas de información en la nube, en organizaciones del sur de Sonora se obtuvieron los siguientes hallazgos.

Las organizaciones están conscientes de que necesitan utilizar las tecnologías de información, y una buena opción para reducir costos es el uso del software en la Nube, esto debido a los grandes beneficios encontrados en su uso. Dentro de los mayores beneficios por los cuales estas empresas se inclinan hacia el uso de "la nube" están que los sistemas de información ayudan a la gerencia en la toma de decisiones, aumenta los controles operativos, apoya en la detección de incidencias, entre otros. (Ver tabla 1).

Tabla 1. Beneficios esperados en las organizaciones del sur de Sonora con la implementación de tecnologías de la información en la nube.

\begin{tabular}{|l|l|}
\hline Organizaciones & Beneficios \\
\hline
\end{tabular}




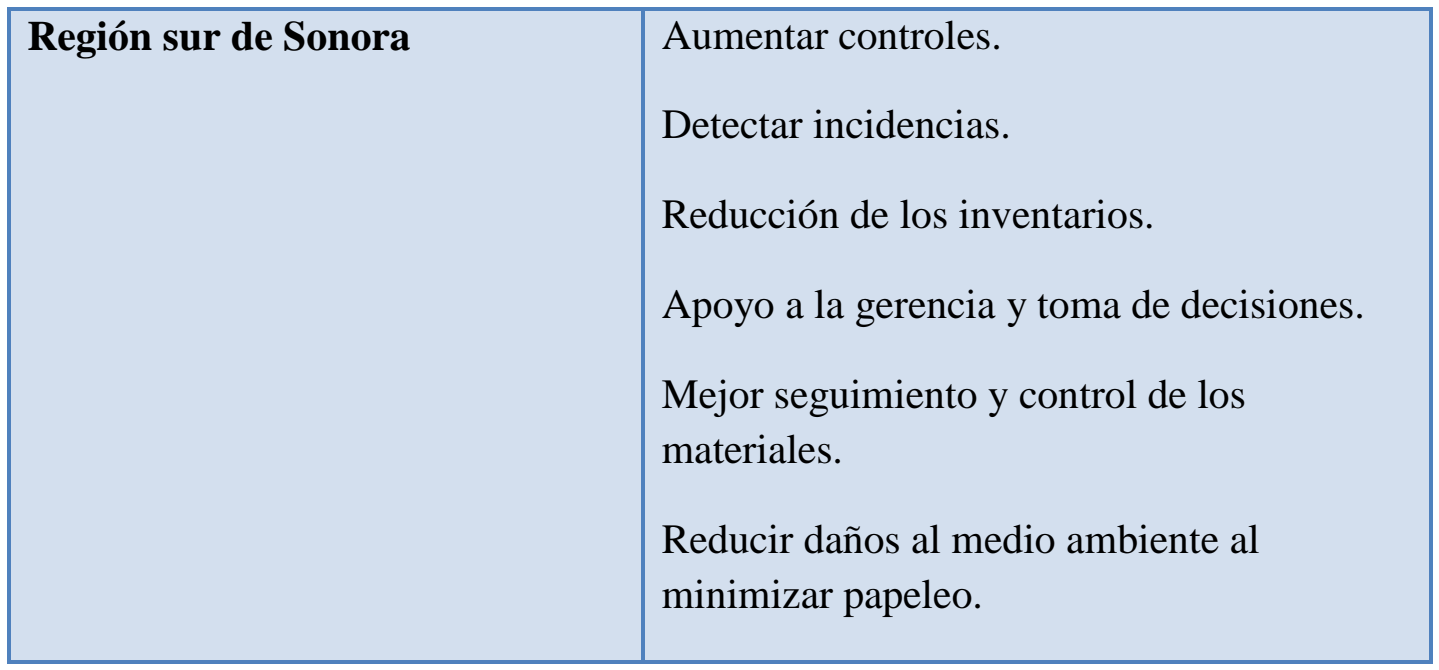

Fuente: información directa

Las empresas del sur de Sonora están preocupadas por estar a la vanguardia, de tal manera que están dispuestas a implementar sistemas de información en la nube para sus empresas, lo cual representa una oportunidad en un terreno poco explorado; se requiere romper con esquemas antiguos. Es difícil, sin embargo, el desarrollo tecnológico obliga a estar a la vanguardia.

Se encontró que dentro de los principales beneficios de implementar Software en la Nube están: disponer de acceso a los datos desde cualquier dispositivo, sin necesidad de estar dentro de la misma ubicación; ahorrar en implementaciones de Software y Hardware; mejoría de controles operativos (ver tabla 2). Los sistemas novedosos de software pueden ayudar a las organizaciones a lograr ventaja sobre sus competidores al permitirles tener acceso a la información y realizar mejores análisis en menos tiempo.

Tabla 2. Principales beneficios de la implementación de Software en la Nube.

\section{Acceso a los datos desde cualquier sitio con}


Revista Iberoamericana de las Ciencias Computacionales e Informática ISSN: 2007-9915

\begin{tabular}{|c|c|}
\hline & Internet \\
\hline 2. & Ahorro en Software y Hardware \\
\hline 3. & Ahorro en mantenimiento técnico \\
\hline 4. & Mayor equilibrio en las operaciones \\
\hline 5. & $\begin{array}{l}\text { Mejora en controles operativos con apoyo de la } \\
\text { Nube }\end{array}$ \\
\hline 6. & $\begin{array}{l}\text { Compartir información evitando la duplicidad de } \\
\text { datos. }\end{array}$ \\
\hline
\end{tabular}

Fuente: Información directa.

A pesar de las grandes ventajas que se obtienen de las tecnologías de información, existe un desconocimiento de los beneficios que pueden obtener las organizaciones al implementar controles operativos en la nube. A continuación se describen cada uno de los principales beneficios encontrados.

1. Facilidad de acceso a la información.

Gracias a la nube es posible acceder a la información desde cualquier lugar con acceso a Internet, solo con tener la dirección Web de la aplicación, contar con un usuario y contraseña. De esta manera, los directivos y administradores de las empresas pueden monitorear las actividades y transacciones que la empresa realiza sin necesidad de pedir la información a terceros, ser procesada y analizada para poder llegar a manos de quien la solicita. De tal manera que utilizando "la Nube", la información siempre está a la mano y en el tiempo que se requiere, accediendo a ella desde cualquier dispositivo con navegador.

2. Beneficios de la centralización de la información 
Revista Iberoamericana de las Ciencias Computacionales e Informática ISSN: 2007-9915

La información se almacena en bases de datos en un servidor con Internet, así se evita el problema de tener la información guardada en cada computadora, es decir, toda la información está almacenada en un solo lugar. El Software también está instalado en un solo sitio, evitando de esa manera tener que instalar el Software de manera estacional en cada computadora que requiera acceso a la información.

3. Ahorros en Hardware y Software

Al estar la información y la aplicación almacenadas en servidores remotos, ya no es necesario instalar servidores locales. Los costos los deben solventar los proveedores, quienes se preocupan por las actualizaciones técnicas, se encargan también de cambiar a un servidor más robusto si es necesario, inclusive sin que los clientes finales lo puedan persuadir. Asimismo, deben mantener el Software de los servidores actualizados para evitar problemas de ataques cibernéticos; el cliente final no se dará cuenta de tales actualizaciones, ni se producirán efectos negativos en los procesos organizacionales.

4. Ahorros en mantenimiento técnico

Los costos de mantenimiento de servidores irán por parte del proveedor del servicio, de esta manera, las empresas que no tienen la solvencia suficiente para tener acceso a los beneficios de los sistemas de información, con el uso de la nube se ahorran estos costos.

5. Mejora de controles operativos 
Revista Iberoamericana de las Ciencias Computacionales e Informática ISSN: 2007-9915

Los controles operativos suelen preocupar a los directos, ya que la mayoría de los Software solo cubren la parte administrativa, sin embargo, con el uso de la nube se facilita la implementación de controles operativos y la alimentación al sistema desde el campo de trabajo, mismo que casi siempre es fuera de la oficina. Con la nube se abren nuevas puertas al ser desarrollados en plataformas en línea, pueden alimentarse de información desde el campo en tabletas con tecnología $3 \mathrm{~g}$, y monitorear la información desde las oficinas por los administradores.

6. Ahorro en tiempos de desarrollo e implementación

La mayoría de las empresas solicita la instalación de Software porque tienen problemas en la organización. Piensan resolver los problemas organizacionales con las tecnologías de información, sin embargo, cuando se trata de Software estacional es mayor el tiempo de implementación, puesto que se necesita también de verificación de compatibilidad con dispositivos y computadoras. Por el contrario, con Software en la Nube solo se requiere un navegador Web para poder tener acceso a la aplicación.

7. Mayor compromiso por parte de los proveedores del servicio

Los servicios en la Nube han llegado para cambiar el paradigma de los negocios del Software. En la mayoría de estos servicios se utilizan pólizas para pagos mensuales, a diferencia del Software estacional que se realiza en una sola exhibición la mayoría de las ocasiones. Este nuevo modelo de negocio crea un mayor compromiso por el proveedor de mantener el servicio seguro y en línea las 24/7. 
8. Compartir archivos

Cuando la información está rezagada, las empresas se estancan. Uno de los principales beneficios de la nube es que propician el hecho de compartir la información, sin embargo, se toman ciertas medidas, siendo una de ellas la jerarquización de roles y niveles de acceso, medida que se realiza con apoyo de los directivos de la empresa.

9. Impacto ambiental

Una de las grandes preocupaciones a nivel mundial es el impacto ambiental causado por las tecnologías de información, pero existe un punto a favor de la empresas que utilizan la Nube, un ejemplo es cuando se implementa Ofimática en la Nube, ya que se reducen costos al no ser necesario imprimir pues los oficios electrónicos son compartidos de manera automática con las personas de la organización que así lo requieran.

10. Seguridad de la información

A diferencia de la creencia común, los sistemas en la Nube son más seguros que los Software estacionales, ya que al estar la información almacenada en Servidores y estos alojados de forma física en lugares remotos y en la mayoría de las ocasiones con sistemas espejo, es poco probable que la información se pierda. Como ya se mencionó, está el compromiso de los proveedores de asegurar la integridad de la 
Revista Iberoamericana de las Ciencias Computacionales e Informática ISSN: 2007-9915

información, así como dar las recomendaciones necesarias para evitar problemas de seguridad técnica y de ingeniería social.

\section{Conclusiones y recomendaciones}

Con el auge de las tecnologías de información en los últimos años, estas han cambiado la forma como operan las organizaciones actuales, mismas que han tenido que adaptarse a las nuevas tecnologías, de tal manera que invirtiendo en tecnologías de información logren minimizar costos.

Las tecnologías de información se han vuelto una necesidad en las empresas, loc aul se refleja al ser implementadas en la mayoría de las organizaciones del Mayo, sin embargo, existe una nueva tendencia que ha llegado a cambiar los paradigmas del intercambio de la información, y a salvar a las pequeñas empresas que aún no cuentan con suficiente dinero para realizar implementaciones de servidores y así poder almacenar la información que requieren para la toma de decisiones. La "Nube" ofrece grandes beneficios a las empresas con poca inversión: el análisis de datos se puede hacer en cualquier momento, cualquier lugar y desde cualquier dispositivo con acceso a Internet, proceso útil para los ejecutivos y dueños de empresas, quienes pueden estar al pendiente de ellas con tan solo un click.

Recomendaciones para explotar los beneficios de los sistemas de información en la nube y reducir costos. Demostrar a las empresas los beneficios de la utilización de sistemas ERP y CRM en la nube, como Planificador de Recursos de la empresa. 
Revista Iberoamericana de las Ciencias Computacionales e Informática ISSN: 2007-9915

El ERP es un sistema integral de gestión empresarial que está diseñado para modelar y automatizar la mayoría de procesos en la empresa (área de finanzas, comercial, logística, producción, etcétera). Su misión es facilitar la planificación de todos los recursos de la empresa, cuyos beneficios serán controlar costos, inventarios, entre otros, de una manera unificada.

Solo un sistema para manejar muchos de sus procesos comerciales, la integración entre las funciones de las aplicaciones, reduce los costos de gerencia e incrementa el retorno de inversión.

Beneficios de CRM: los sistemas informáticos que manejan CRM mejoran el desempeño de las organizaciones que tienen una relación con clientes de manera muy precaria, de tal manera que estas implementaciones crean un círculo de confianza entre las empresas y los clientes, formando un lazo que fortalece su relación. Así, las organizaciones conocen bien a sus clientes y pueden ofrecerles un servicio personalizado, corriendo menos riesgo de perder clientes importantes y encontrar clientes potenciales.

Las soluciones en la nube son las más efectivas para empresas que no requieren contar con un departamento de sistemas. El hardware, las instalaciones físicas, el desarrollo de software y el mantenimiento son aspectos de los cuales las organizaciones dejarían de preocuparse si aplicaran soluciones en la nube, lo que les permitiría enfocar sus esfuerzos en mejorar sus procesos de producción o calidad. 
Revista Iberoamericana de las Ciencias Computacionales e Informática ISSN: 2007-9915

Las organizaciones muchas veces sin saberlo hacen uso de servicios en la nube de manera constante, por ejemplo, es habitual consultar el correo electrónico desde una página Web como es el caso de gmail o Facebook.

Ventajas de la nube: acceso desde cualquier sitio y con varios dispositivos. Tus programas y archivos están en la nube, con lo que te basta una conexión a Internet para acceder a ellos y usarlos de modo remoto.

Lo anterior se puede hacer desde una computadora fija, una laptop, una Tablet, una PC, un iPod, un Smartphone. Todo el software está en un solo sitio, eso evita tener que instalar Software en las computadoras, laptop o todos y cada uno de los múltiples equipos de las organizaciones. Para ventaja de las empresas, no solo evita instalar el software, sino que también resulta innecesario hacer actualizaciones en cada computadora que tenga instalada cierta aplicación que requiera. De manera paralela, se minimizan problemas de compatibilidad. Para que la metodología de la nube funcione en una organización, el único requisito es que los dispositivos tengan instalado un navegador de Internet con el que puedan trabajar en ella. Otro factor fundamental es contar con servicio de internet.

Aun así predominan las ventajas tanto para los proveedores de servicios en la nube como para las organizaciones. Al implementarse la nube se ahorra en software y hardware. En la nube, un mismo programa lo comparten muchos usuarios, sin necesidad de comprar una copia individual para cada uno de ellos. De esta manera, se minimizan gastos de hardware, software, mantenimiento de software y grandes gastos en dispositivos de almacenaje de 
Revista Iberoamericana de las Ciencias Computacionales e Informática ISSN: 2007-9915

información. Al final, se tienen organizaciones menos preocupadas por la infraestructura tecnológica y más enfocadas en el aprovechamiento del software que la nube les provee.

\section{BIBLIOGRAFIA}

Bell, J. (2002). Cómo hacer tu primer trabajo de investigación. Guía para investigadores en Educación y ciencias sociales. Barcelona: Ed. Gedisa.

ConceiçãoMenezes, P. A., \& González-Ladrón-de-Guevara, F. (2010). Maximización de los beneficios de los sistemas ERP. JISTEM: Journal of Information Systems and TechnologyManagement , 7 (1), 5-32.

Gutiérrez, A. (2010). Computación en la Nube. Revista Ingeniería Primero. Universidad Rafael Landivar de Guatemala.

Laudon, K. C., \& Laudon, J. P. (2008). Sistemas de información gerencial: Administración de la empresa digital. D.F., México: Prentice Hall Hispanoamericana.

O’Brien, J. (2001). Sistemas de información gerencial. Colombia: Editorial McGraw-Hill Interamericana, S.A.

Rodríguez, Roció(2012). Antecedentes y consecuencias del uso de las NTIC por parte de los vendedores. Tesis, Facultad de Economía y Empresa, Universidad de Murcia.

Somers, M. T., \& Nelson, K. (2004). A taxonomy of players and activities across the ERP project life cycle. Information \& Management, 41, 257-278. 